\title{
Modelado físico de la incidencia de un chorro de aire sobre una superficie de agua ${ }^{(\cdot)}$
}

\author{
J. Solórzano-López*, M.A. Ramírez-Argáez** y R. Zenit***
}

\begin{abstract}
Resumen
El uso de chorros de gas (oxígeno) es fundamental en diversos procesos de aceración, como el Horno Básico de Oxígeno (BOF, por sus siglas en inglés) o en el Horno Eléctrico de Arco (EAF). Estos chorros mejoran la transferencia de calor, masa y cantidad de movimiento en el metal y el mezclado de especies y son muy importantes en la formación de escoria espumosa. En este trabajo, se realizaron mediciones experimentales de las dimensiones de la cavidad formada en la superficie líquida por la incidencia de un chorro, así como también se obtuvieron vectores de velocidad en la zona aledaña a dicha cavidad. Éstas, se midieron usando una cámara de alta velocidad, mientras que los mapas vectoriales se obtuvieron por la técnica PIV (Particle Image Velocimetry). Las velocidades y las dimensiones de las cavidades se determinaron como función de las principales variables de proceso: flujo de gas, distancia entre tobera y superficie libre, diámetro de tobera y ángulo de lanza. Las dimensiones de la cavidad se procesaron estadísticamente, como función de las variables de proceso y como función de los números adimensionales que lo gobiernan. En este caso, el número de Froude junto con el de Weber controlan la geometría de la depresión. El flujo en el seno del líquido es principalmente afectado por el flujo de aire, la altura y el ángulo de la lanza.
\end{abstract}

Palabras clave $\quad$ Modelado físico; Flujo de fluidos; Chorros de gas; Superficie libre; PIV.

\section{Physical modeling of the impingement of an air jet on a water surface}

\begin{abstract}
The use of gas jets (oxygen) plays a key role in several steelmaking processes as in the Basic Oxygen Furnace (BOF) or in the Electric Arc Furnace (EAF). Those jets improve heat, mass and momentum transfer in the liquid metal, mixing of chemical species enhancing and govern the formation of foaming slag. In this work experimental measurements were performed to determine the dimensions of the cavity formed at the liquid free surface caused by a gas jet impinging on it; also velocities vectors were measured in the zone affected by the gas jet. Cavities were measured from images from high speed camera and the vector maps were obtained with a Particle Image Velocimetry (PIV) technique. Both velocities and cavities were determined as a function of the main process variables: gas flow rate, distance of the nozzle from the free surface and lance angle. Cavity dimensions were statistically processed treated as a function of the process variables and also as a function of the proper dimensionless numbers that govern these phenomena. It was found that Weber and Froude numbers govern the cavity geometry. Liquid flow driven by the jet is mainly affected by the air flow rate, lance height and angle.
\end{abstract}

Keywords

Physical modeling; Fluid flow; Gas jet; Free surface; Particle image velocimetry.

\section{INTRODUCCIÓN}

Los chorros de oxígeno se usan, ampliamente, en procesos de aceración como el Horno Básico de Oxígeno (BOF) y el Horno Eléctrico de Arco (EAF). Estos chorros tienen un papel importante en dichos procesos porque controlan la cinética de las reacciones químicas, la formación de escoria espumosa, el mezclado del baño y el fenómeno de salpicado, debido a su intercambio de cantidad de movimiento, calor y masa con el acero fundido ${ }^{[1 \text { y } 2]}$. Para evaluar la transferencia de cantidad de movimiento entre los chorros y el acero líquido, se debe determinar la geometría de las depresiones formadas en la superficie

\footnotetext{
(•) Trabajo recibido el día 9 de Octubre de 2009 y aceptado en su forma final del día 22 de Abril de 2010

${ }^{*}$ Facultad de Química, Departamento de Ingeniería Metalúrgica, Universidad Nacional Autónoma de México, México, D.F., 04510, México. E-mail: xaxni2006@yahoo.com.mx.

${ }^{* *}$ Facultad de Química, Departamento de Ingeniería Metalúrgica, Universidad Nacional Autónoma de México, México, D.F., 04510, México.

E-mail: marco.ramirez@servidor.unam.mx

*** Instituto de Investigaciones en Materiales, Universidad Nacional Autónoma de México, Av. Universidad 3000, Coyoacán, 04510, México, D.F.
} 
libre, así como los perfiles de velocidad causados por la incidencia y los esfuerzos cortantes del chorro, respectivamente.

Tanto la velocidad del líquido como la geometría de la depresión dependen de las variables del proceso, como son flujo de gas, distancia entre la tobera y la superficie libre, ángulo formado entre la lanza y la superficie libre, así como tamaño y número de toberas, tipo de apertura de las mismas, densidad y viscosidad de la fase gas y la fase líquida, entre otras.

Es difícil llevar a cabo mediciones en los procesos industriales que involucran metal fundido ${ }^{[3]}$, por ello, los modelos físicos con agua son una herramienta útil para realizar estudios en el laboratorio, a bajo costo y en condiciones de trabajo seguras. Diversos grupos de investigación han medido el tamaño de la profundidad de la cavidad formada por el chorro incidente en el baño líquido y han publicado sus resultados en forma de correlaciones empíricas del tamaño de la profundidad, como función de los principales parámetros de operación. La tabla I resume las correlaciones reflejadas en la literatura ${ }^{[4]}$.

La mayoría de las correlaciones relacionadas en la tabla I se obtuvieron por medición directa en modelos físicos con agua, excepto aquellas obtenidas por Sharma et al. ${ }^{[3]}$ y por Koria et al..$^{[5]}$, que se realizaron en hornos piloto. Sharma et al. concluyeron que el tamaño de la cavidad es gobernado, mecánicamente, por la fuerza del chorro, mientras que Koria et al. dan más importancia a la distancia existente entre la tobera y la superficie del baño, así como a la presión del gas (incidiendo en una superficie metálica). Qian et al. ${ }^{[6]}$ aportan mediciones de cavidades, en un modelo con agua con una ligera capa superior de un segundo líquido (keroseno o aceite), para simular la presencia de escoria. Subagyo ${ }^{[7]}$ correlacionó con éxito el fenómeno de salpicado con el número de Weber normalizado. Memolli et al. ${ }^{[8]}$ simularon la penetración del chorro en un baño líquido, asumiendo que la cavidad es un paraboloide de revolución. Meidani et al. ${ }^{[9]}$ presentaron, en su trabajo, un modelo que no toma en cuenta los efectos de viscosidad y tensión superficial. La validez de estas correlaciones no puede ser cuestionada; sin embargo, de estas correlaciones no es factible entender cuál es el mecanismo físico que controla el fenómeno.

Por otra parte, el perfil de velocidades en el seno del líquido, inducido por la transferencia de cantidad de movimiento del chorro de gas, es importante en el fenómeno de mezclado y en la cinética de las reacciones gobernadas por la transferencia de masa de las especies químicas involucradas. Muchos trabajos de investigación han modelado, matemáticamente, este intercambio de cantidad de movimiento chorro gaseoso-baño usando técnicas CFD (Computational Fluid Dynamics $^{[10]}$. Se han publicado mediciones de los perfiles de velocidad en el baño causados por la incidencia del chorro, usando la técnica Particle Image Velocimetry

Tabla I. Diferentes correlaciones adimensionales descritas en la literatura para la profundidad de la cavidad como función de las variables principales cuando un chorro de gas incide en la superficie libre del baño líquido ${ }^{[4]}$

Table I. Differents dimensionless reported correlations that describe de cavity depth as process variables function when a gas jet impinges in the free surface of a liquid bath ${ }^{[4]}$

\begin{tabular}{|c|c|c|}
\hline Investigador & Año & Correlación del tamaño de la profundidad \\
\hline Collins y Lubanska & 1954 & $H j=\left(0,53^{*} P^{*} \cos \alpha\right) /\left[\left(\rho_{l}^{*} g^{*} h^{2 *} \sec ^{2} \alpha\right)+19^{*}\left(\rho_{l}^{*} g^{*} P^{2}\right)^{1 / 3}\right]$ \\
\hline Banks y Chandrasekhara & 1963 & $(H j / h)^{*}(1+H j / h)^{2}=(125 / \pi)^{*}\left(P / \rho_{l}^{*} g^{*} h^{3}\right)$ \\
\hline Wakelin & 1966 & $(H j / h)^{*}(1+H j / h)^{2}=\left[\left(2^{*} K_{2}^{2}\right) / \pi\right]^{*}\left(P / \rho_{l}^{*} g^{*} h^{3}\right) ; K_{2}=6,4$ \\
\hline Davenport & 1966 & $(H j / h)^{*}(1+H j / h)^{2}=\left[\left(2^{*} K_{1}^{2}\right) / \pi\right]^{*}\left(P / \rho_{l}^{*} g^{*} h^{3}\right) ; K_{1}=7,6$ \\
\hline Flinn, Pehlke, Glass y Hays & 1967 & $H j^{\prime}=1,5^{*} p_{o}{ }^{* *} d_{t}^{\prime *} h^{\prime 0.5}+1,5$ \\
\hline Chatterjee & 1973 & $H j / h^{*}\left\{1+\left[H j-\left(7^{*} d_{d}\right)\right] / h\right\}^{2}=100^{*} P /\left(p^{*} g^{*} r_{1}^{*} h^{3}\right)$ \\
\hline Koria y Lange & 1987 & $\begin{array}{l}H j / h=4,469^{*}\left[0,7854^{*} 10^{5 *} d_{t}^{2 *} p_{a m b}{ }^{*}\right. \\
\left.\left(1,27^{*} p_{o} / p_{a m b}-1\right)^{*} \cos \alpha /\left(g^{*} \rho_{l}^{*} h^{3}\right)\right]^{0,66}\end{array}$ \\
\hline Qian, Muthasaran y Farouk & 1996 & $(H j / h)^{*}(1+H j / h)^{2}=\left[\left(2^{*} K_{2}^{2}\right) / p\right]^{*}\left[P /\left(r_{l}^{*} g^{*} h^{3}\right) ; K_{2}=7,5\right.$ \\
\hline Kumagai y Iguchi & 2001 & 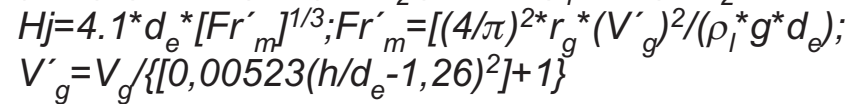 \\
\hline $\begin{array}{l}\text { Olivares, Elías, Sánchez, } \\
\text { Cruz y Morales }\end{array}$ & 2002 & $\left(H j^{*} h / d_{t}^{2}\right)=1,508^{\star} F r^{0,714} ; F r=\left(V_{g}{ }^{2} / d_{t}^{*} g\right)^{\star}\left[\rho_{g}\left(\rho_{\Gamma} \rho_{g}\right)\right]$ \\
\hline
\end{tabular}


(PIV). Gu ${ }^{[11]}$ realizó las mediciones para validar un modelo matemático desarrollado en CFD. Schwarz ${ }^{[12]}$ desarrolló, también, un modelo matemático usando CFD, pero no validó sus resultados numéricos con resultados experimentales. Peaslee ${ }^{[13]}$ trabajó con chorros supersónicos en la determinación de la geometría de la cavidad y usó sus mediciones experimentales para validar los resultados obtenidos con CFD.

En el presente trabajo, se realizaron mediciones experimentales de la geometría de la cavidad formada por la incidencia de un chorro de aire sobre una superficie libre, a través del uso de cámaras de alta velocidad. Estas, se presentan junto con los perfiles, medidos con la técnica PIV, de velocidad del líquido en movimiento por la transferencia de cantidad de movimiento del chorro y se analizan como función de las principales variables de proceso, como flujo de gas, distancia entre tobera y superficie libre, ángulo de la tobera con respecto a la superficie libre y diámetro de la tobera. Este trabajo se distingue de los anteriores porque presenta, de manera conjunta, mediciones de los perfiles de velocidad y geometría de la cavidad formada (ancho o diámetro y profundidad), así como correlaciones obtenidas estadísticamente, que describen la geometría de la cavidad como función de las principales variables de proceso y, también, como función de los principales números adimensionales involucrados en el mismo.

\section{PROCEDIMIENTO EXPERIMENTAL}

El modelo físico se construyó con acrílico transparente, formando un cilindro de $0,3 \mathrm{~m}$ de altura y con diámetro interno de $0,2 \mathrm{~m}$, cubierto con un tanque rectangular para evitar la distorsión por curvatura. La lanza se fabricó con tubo de cobre de 0,012 m de diámetro interno, con toberas cilíndricas intercambiables de diversos diámetros fabricadas, también, en cobre. Para el flujo de aire, se usaron un compresor y un medidor de flujo, mientras que un sistema de PIV que incluye luces láser y una cámara de alta velocidad se utilizó para capturar los mapas de velocidad en el líquido. El arreglo experimental se muestra en la figura 1.

La determinación de la profundidad de la cavidad se hizo mediante una Cámara de Alta Velocidad (HSC, por sus siglas en Inglés) Red Lake Motion Scope, utilizada a 500 disparos por segundo, tiempo de exposición de $1 / 500$ y frecuencia de 2 segundos. Las imágenes digitales obtenidas tuvieron una resolución de 9 pixeles/mm y las mediciones se hicieron directamente imagen por imagen, usando una reglilla a escala. Estos resultados se procesaron estadísticamente, aplicando regresión lineal multivariable con un software comercial.

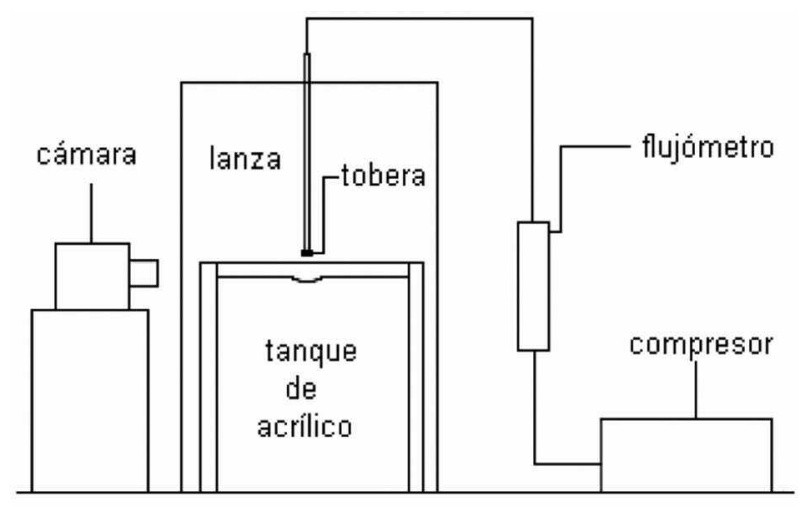

Figura 1. Arreglo experimental usado para medir la geometría de la depresión o cavidad así como los perfiles de velocidad causados por la incidencia del chorro sobre la superficie libre líquida del modelo.

Figure 1. Experimental setup used in cavity geometry and velocity profiles measurements in the gas jet impinging on the model liquid free surface.

Un equipo PIV (Dantec Dynamics) con software de análisis de imágenes (Flow Manager 4.5) se usó para obtener los mapas de velocidad en la región del baño afectada por el chorro. Se adicionaron al agua esferas de vidrio con la superficie metalizada para que actuasen como marcadores, necesarios para llevar a cabo las correlaciones cruzadas que se requieren para medir los perfiles de velocidad; el porcentaje de traslape fue del $50 \%$ en la malla usada para tal propósito. Las imágenes se enmascararon (es decir, se cubrieron selectivamente con rectángulos negros) para evitar analizar las zonas donde no había líquido, cerca del punto de incidencia del chorro gaseoso. Los perfiles promedio de velocidad se obtuvieron, finalmente, para cada conjunto de condiciones experimentales. Los experimentos se llevaron a cabo por triplicado para proveer significancia estadística. Las variables consideradas en este estudio se presentan en la tabla 2, basada en la escala de Nordquist et al..$^{[4]}$.

Es necesario puntualizar que una nueva variable $(z)$ se introduce en la descripción y corresponde a la distancia de la tobera a la superficie libre del líquido. Se determinó, trigonométricamente, usando la altura de lanza y el ángulo de la misma. La figura 2 muestra el arreglo geométrico para calcular $z$. Esta variable se usó para obtener las correlaciones reportadas en este trabajo en lugar del ángulo y la altura de lanza como variables independientes.

Es preciso recalcar que la geometría de la depresión formada depende de un balance de fuerzas que definen sus dimensiones. Estas fuerzas son: la fuerza de inercia que tiene el jet de gas al pegar en la superficie 
Tabla II. Condiciones para cada experimento

Table II. Experimental conditions for each experiment

\begin{tabular}{|c|c|c|c|}
\hline $\begin{array}{l}\text { Diámetro de } \\
\text { tobera }(\mathrm{m})\end{array}$ & $\begin{array}{l}\text { Ángulo } \\
\text { de lanza } \\
\text { (grados) }\end{array}$ & $\begin{array}{c}\text { Flujo de } \\
\text { gas } \\
\left(\mathrm{m}^{3} / \mathrm{s}\right)\end{array}$ & $\begin{array}{c}\text { Altura } \\
\text { de lanza } \\
\text { (m) }\end{array}$ \\
\hline $1,58 \times 10^{-3}$ & 60 & $1 \times 10^{-3}$ & 0,02 \\
\hline \multirow[t]{4}{*}{$3,17 \times 10^{-3}$} & 75 & $1,3 \times 10^{-3}$ & 0,05 \\
\hline & 90 & $1,6 \times 10^{-3}$ & 0,08 \\
\hline & & $2 \times 10^{-3}$ & 0,1 \\
\hline & & & 0,02 \\
\hline \multirow[t]{4}{*}{$1,58 \times 10^{-3}$} & 60 & $1,3 \times 10^{-3}$ & 0,05 \\
\hline & & & 0,08 \\
\hline & & & 0,1 \\
\hline & & $1 \times 10^{-3}$ & \\
\hline \multirow[t]{3}{*}{$1,58 \times 10^{-3}$} & 60 & $1,3 \times 10^{-3}$ & 0,05 \\
\hline & & $1,6 \times 10^{-3}$ & \\
\hline & & $2 \times 10^{-3}$ & \\
\hline \multirow[t]{3}{*}{$1,58 \times 10^{-3}$} & 60 & $1,3 \times 10^{-3}$ & 0,05 \\
\hline & 75 & & \\
\hline & 90 & & \\
\hline $1,58 \times 10^{-3}$ & 60 & $1,3 \times 10^{-3}$ & 0,05 \\
\hline $3,17 \times 10^{-3}$ & & & \\
\hline
\end{tabular}

libre y que provoca la depresión; la fuerza de gravedad que actúa sobre el líquido y que se opone a la formación

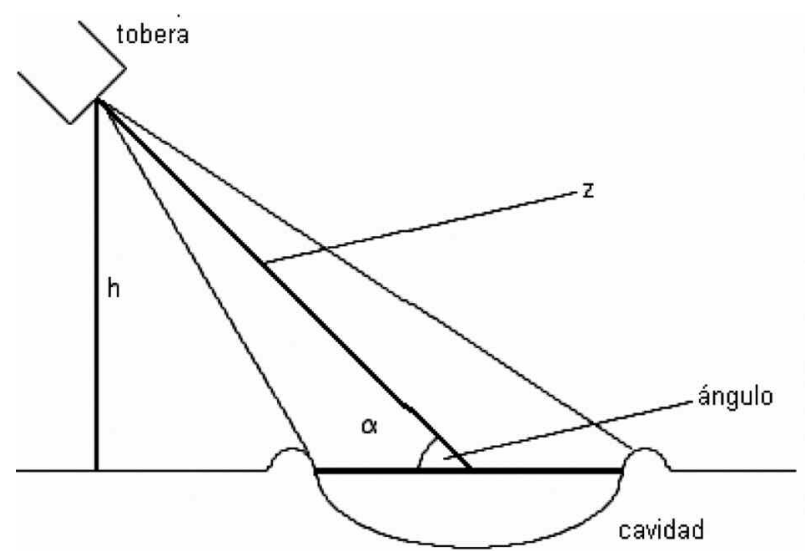

Figura 2. Distancia, $z$, considerada desde la tobera a la superficie del baño y que se obtiene por trigonometría a partir de la altura de lanza, $h$, y del ángulo de la lanza, $\alpha$.

Figure 2. Distance z from nozzle tip to surface bath obtained by trigonometry from the lance height, $\mathrm{h}$, and the lance angle, $\alpha$. de la depresión; y la fuerza de tensión superficial entre el líquido y el gas y que, también, se opone a la deformación. Estas fuerzas están contenidas en los números de We, Re y Fr que se presentan a continuación:

$$
\begin{gathered}
W e=\left(\rho_{g} * R_{o} * V_{g}^{2}\right) / \sigma \\
\operatorname{Re}=\left(L * V_{g} * \rho_{g}\right) / \mu_{g} \\
F r=V_{g}^{2} /(g * H j)
\end{gathered}
$$

\section{RESULTADOS Y DISCUSIÓN}

\subsection{Dimensiones de la cavidad}

Midiendo experimentalmente el ancho o diámetro y la profundidad de la cavidad (Fig. 3) se pudo determinar que algunas variables afectan, más que otras, las dimensiones de esta.

Con respecto al ancho de la cavidad, el flujo de gas del jet tiene un efecto directamente proporcional

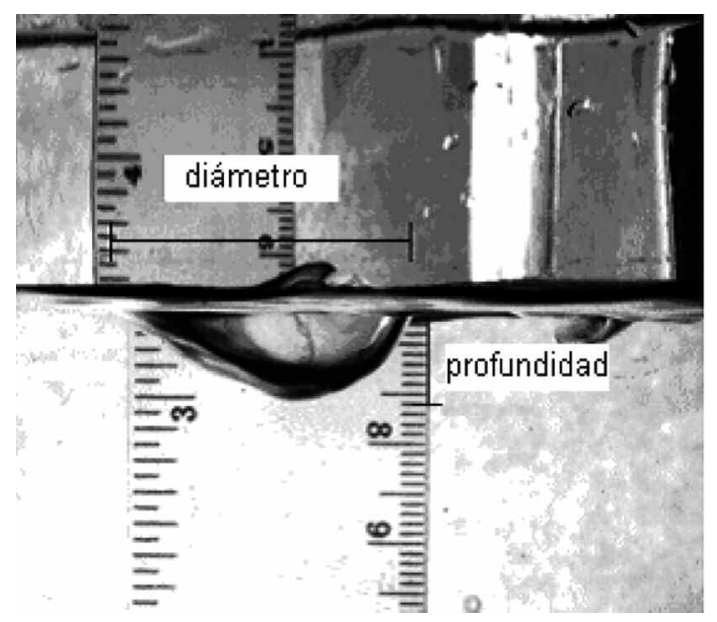

Figura 3. Fotografía típica de una cavidad formada en la superficie libre por la incidencia del chorro en la superficie libre del baño. Se indican el ancho o diámetro y la profundidad.

Figure 3. Typical photograph of a cavity formed on a free surface due to the impingement of a gas jet. The width and depth are indicated in it. 
mientras que la distancia, $z$, y el diámetro de tobera no muestran un efecto significativo.

Por otro lado, la variable, $z$, tiene un efecto inversamente proporcional en la profundidad de la cavidad, mientras que el flujo de gas es directamente proporcional. El diámetro de la tobera no presenta un efecto significativo en la profundidad de la cavidad. La figura 4 muestra algunas cavidades típicas formadas por la incidencia del chorro a diferentes condiciones experimentales. En la figura 4 a), el

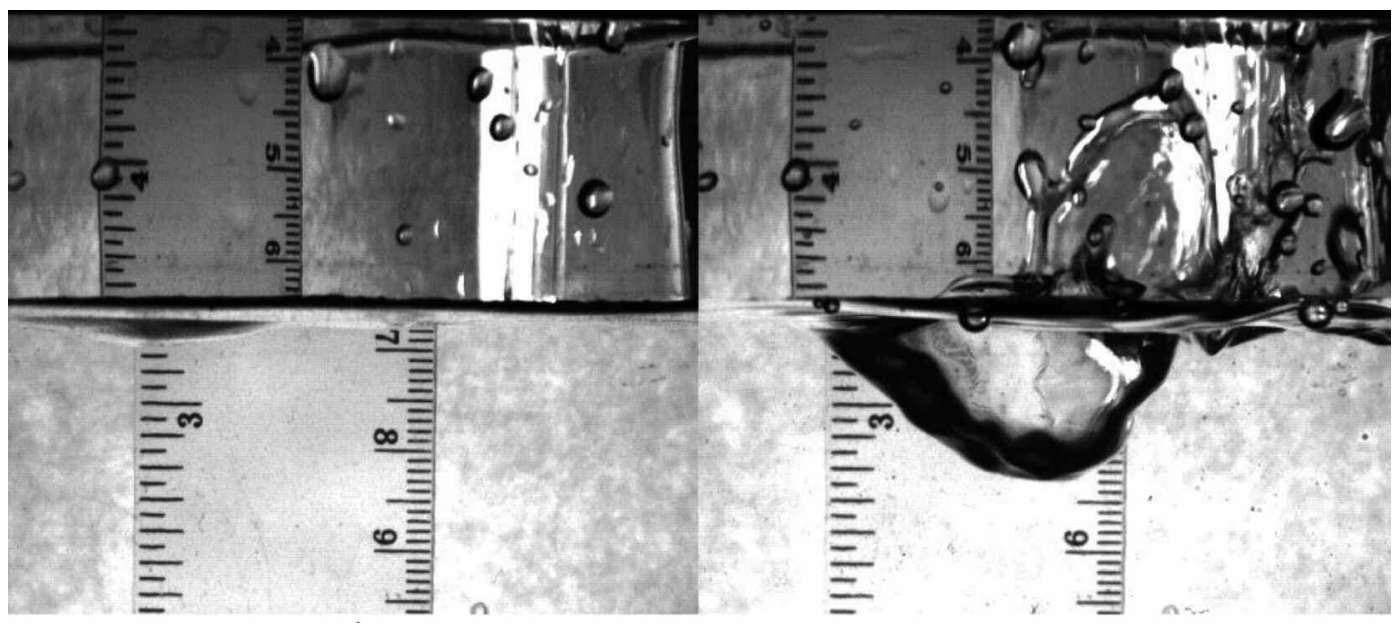

a)

b)

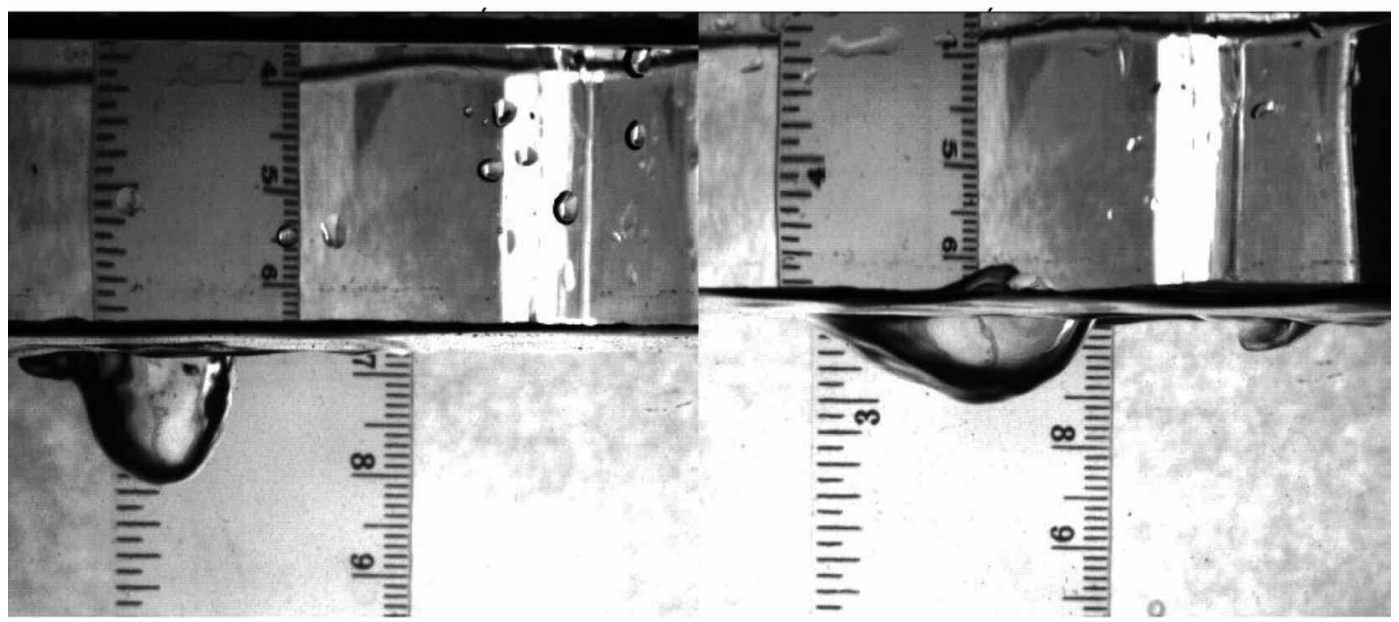

c)

d)

Figura 4. Efecto del chorro gaseoso sobre la geometría de la cavidad. Las condiciones experimentales son: a) diámetro de tobera de $1,58 \times 10^{-3} \mathrm{~m}$, flujo de gas de $1,3 \times 10^{-3} \mathrm{~m}^{3} / \mathrm{s}$, ángulo de lanza de $60^{\circ}$ y altura de lanza $(h)$ de $0,1 \mathrm{~m}$; b) diámetro de tobera de $1,58 \times 10^{-3} \mathrm{~m}$, flujo de aire de $2 \times 10^{-3} \mathrm{~m} / \mathrm{s}$, ángulo de lanza de $60^{\circ}$ y altura de lanza $(h)$ de $0,05 \mathrm{~m}$; c) diámetro de tobera de $1,58 \times 10^{-3} \mathrm{~m}$, flujo de gas de $1,3 \times 10^{-3} \mathrm{~m}^{3} / \mathrm{s}$, ángulo de lanza de $90^{\circ}$ y altura de lanza $(h)$ de $0,05 \mathrm{~m}$; d) diámetro de tobera de $3,17 \times 10^{-3} \mathrm{~m}$, flujo de gas de $1,3 \times 10^{-3} \mathrm{~m}^{3} / \mathrm{s}$, ángulo de lanza de $60^{\circ} \mathrm{y}$ altura de lanza $(h)$ de $0,05 \mathrm{~m}$.

Figure 4. Effect of the gas jet on cavity geometry. Complete experimental conditions are: a) nozzle diameter $1.58 \times 10^{-3} \mathrm{~m}$, gas flow rate $1.3 \times 10^{-3} \mathrm{~m}^{3} / \mathrm{s}$, lance angle of $60^{\circ}$ and lance height (h) $0.1 \mathrm{~m}$; b) nozzle diameter $1.58 \times 10^{-3} \mathrm{~m}$, gas flow rate $2 \times 10^{-3}$ $\mathrm{m}^{3} / \mathrm{s}$, lance angle $60^{\circ}$ and lance height (h) $0.05 \mathrm{~m}$; c) nozzle diameter $1.58 \times 10^{-3}$, gas flow rate $1.3 \times 10^{-3} \mathrm{~m}^{3} / \mathrm{s}$, lance angle $90^{\circ}$ and lance height (h) $0.05 \mathrm{~m}$; d) Nozzle diameter $3.17 \times 10^{-3} \mathrm{~m}$, gas flow rate $1.3 \times 10^{-3} \mathrm{~m}^{3} / \mathrm{s}$, lance angle $60^{\circ}$ and lance height (h) $0.05 \mathrm{~m}$. 
tamaño de la cavidad es el más pequeño, dado que el chorro recorre una gran distancia, $z$, y el flujo de gas es mínimo. En la figura 4 b), el flujo de gas se incrementa hasta $2 \times 10^{-3} \mathrm{~m}^{3} / \mathrm{s} \mathrm{y}$, consecuentemente, la profundidad se incrementa. En la figura $4 \mathrm{c}$ ), con una posición vertical de la lanza $\left(90^{\circ}\right)$, el ancho disminuye pero la profundidad se incrementa al máximo debido a que la cantidad de movimiento es perpendicular a la superficie del baño, con lo que la penetración del chorro se maximiza. Finalmente, en la figura 4 d), el diámetro de la tobera se duplica con respecto a los casos anteriores y las dimensiones de la cavidad son moderadas en cuanto a su ancho y profundidad.

Se llevó a cabo un análisis de las dimensiones de la cavidad, procesando estadísticamente los datos para correlacionar la geometría de la cavidad con las variables de proceso exploradas en este trabajo. Se aplicó una regresión lineal multivariable que dio por resultado las siguientes expresiones para el ancho (a) y la profundidad $(H j)$ de la cavidad como función de las variables de proceso:

$$
\begin{gathered}
a=0,015+9,225^{*} f\left(r^{2}=0,529\right) \\
H j=0,006-0,136^{*} z+9,38^{*} f\left(r^{2}=0,861\right)
\end{gathered}
$$

donde, es el ancho de la cavidad en $\mathrm{m}, H \mathrm{H}$ es la profundidad de la cavidad en $\mathrm{m}, f$ es el flujo de gas en $\mathrm{m}^{3} / \mathrm{s}$, y es la distancia total de la tobera al baño en $\mathrm{m}$.

Se puede apreciar que el ancho de la cavidad solo depende del flujo de gas, en tanto que la profundidad de la cavidad depende tanto de la distancia entre la tobera y el baño como del flujo de gas. Analizando los valores de los coeficientes, el flujo de gas es la variable que afecta en mayor medida la geometría de la cavidad. De acuerdo con estos resultados, ni el ancho ni la profundidad de la cavidad dependen del diámetro de la tobera. El significado de las ecuaciones (4) y (5) es puramente estadístico a través de un análisis de regresión multivariable y sólo son válidas en el rango de condiciones experimentales probadas en este trabajo. Desde luego que la forma de la cavidad depende de la inercia con la que el jet golpea la superficie libre y esta depende a su vez de la distancia $z$, pero para nuestras condiciones experimentales no es estadísticamente significativo el efecto de $\boldsymbol{z}$ sobre el ancho.

La figura 5 muestra la dependencia del ancho de la cavidad contra el flujo de gas. En esta gráfica se representa el juego completo de experimentos. Se puede ver que un incremento en el flujo de gas produce un incremento en el ancho de la cavidad. Las cajas y los percentiles representan el grado de disper-

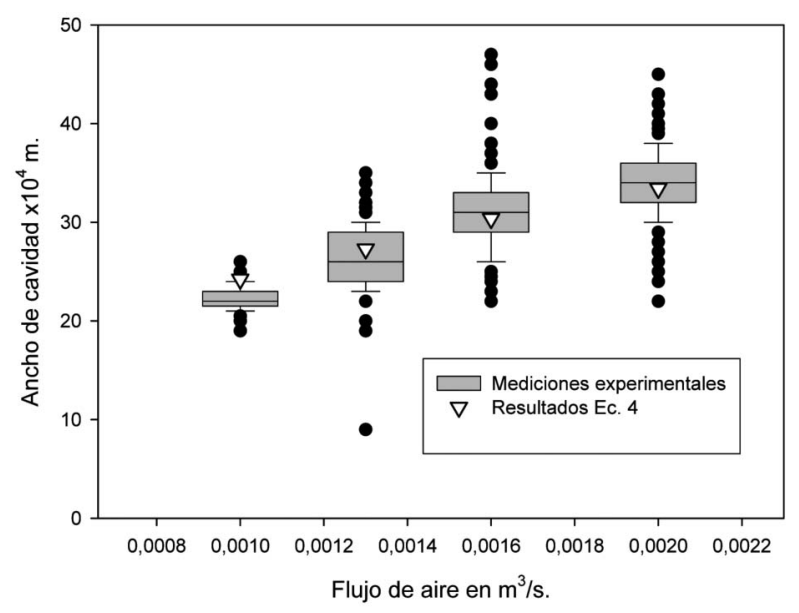

Figura 5. Ancho o diámetro de la cavidad como función del flujo de gas del chorro. Los triángulos blancos son predicciones de la ecuación (4), las cajas y los percentiles representan la desviación estándar del 90 y $95 \%$ de los datos, la línea negra dentro de las cajas es el promedio de los datos y los puntos negros son las mediciones individuales que cayeron fuera del $95 \%$.

Figure 5. Cavity width or diameter as a function of the gas flow rate. White triangle are predictions by equation (4), boxes and percentiles represent standard deviation of 90 and $95 \%$ of data, black line inside boxes is the data mean and black dots are the individual measurements that lied out of the $95 \%$ of data.

sión del 90 y $95 \%$, respectivamente, para cada flujo de gas. La línea en las cajas es el promedio, los puntos negros son las mediciones que cayeron fuera del $95 \%$ del total de datos (del orden de mil fotografías por experimento) y los triángulos blancos representan las predicciones del modelo estadístico, ecuación (4). La figura 5 muestra que el modelo predice correctamente el ancho de la cavidad dependiente del flujo de gas ya que las predicciones son cercanas al promedio de los datos para cada flujo. Se puede apreciar que cuando el flujo de gas se incrementa, también se incrementa la dispersión estadística porque la forma de la cavidad es afectada por inestabilidades asociadas a la turbulencia y las salpicaduras.

Las figuras 6 a) y 6 b) presentan la profundidad de la cavidad como función del flujo de gas y de la distancia entre el baño y la tobera, respectivamente. Como se esperaba, el flujo de gas incrementa la profundidad de la depresión. Por otro lado, un incremento en la distancia entre el baño y la tobera reduce la profundidad de la cavidad. Esta última depende de la magnitud de la cantidad de movimiento 


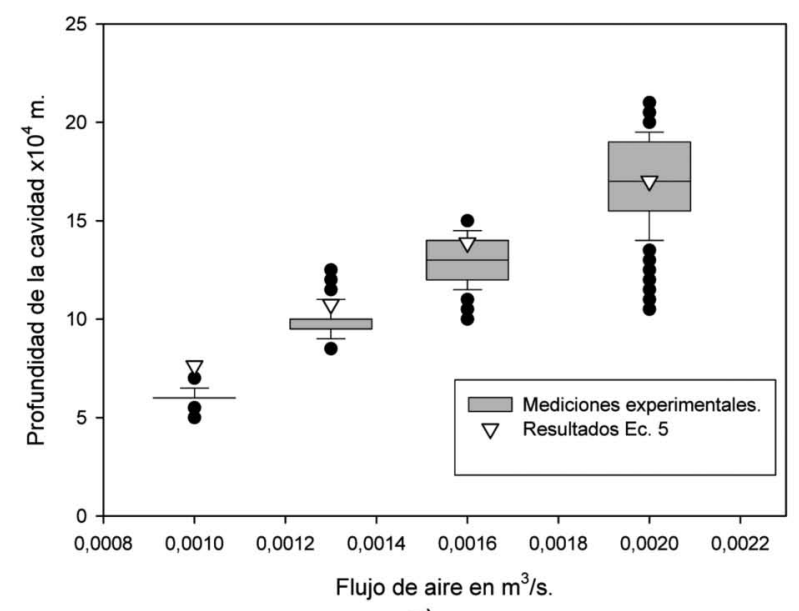

a)

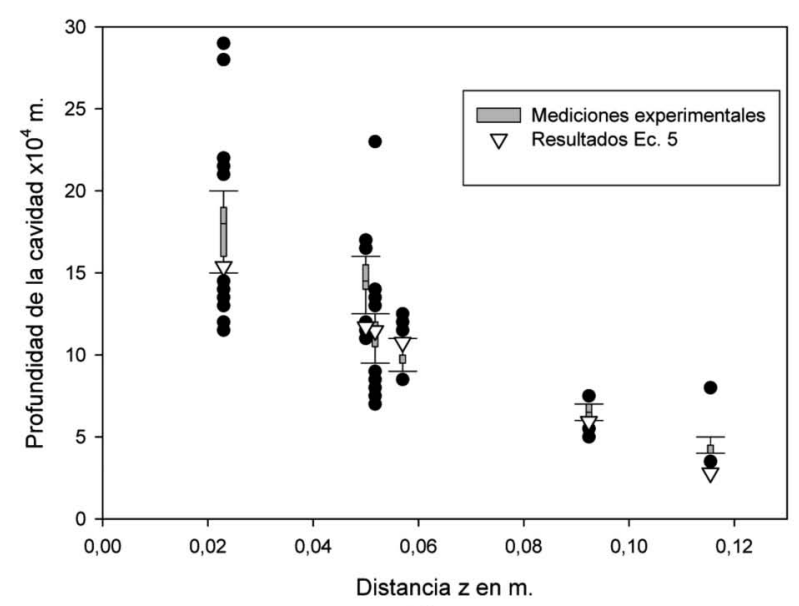

b)

Figura 6. Profundidad de la cavidad como función de: (a) flujo de gas y (b) distancia, $z$, de la tobera al baño. Los triángulos blancos son las predicciones de la ecuación 5 , las cajas, los percentiles, la línea negra dentro de las cajas y los puntos negros representan lo mismo que en la figura 5.

Figure 6. Cavity depth as a function of: (a) gas flow rate and (b) nozzle to bath distance, $\mathrm{z}$. White triangle are predictions by equation 5, boxes, percentiles, black line inside boxes and black dots represent the same as in the figure 5 .

vertical que el chorro transmite a la superficie líquida. Esta cantidad de movimiento vertical es función del flujo de aire, ángulo de la lanza y altura de la misma. Es interesante notar que la dispersión de las medidas de la profundidad de la cavidad se incrementan al incrementarse la cantidad de movimiento del chorro, así como al incrementarse el flujo de gas (Fig. 6 a)) o al disminuir la distancia entre la superficie entre el baño y la tobera (Fig. 6b)), ya que las inesta- bilidades se vuelven más importantes (como se aprecia en la figura 4 b)).

Para obtener una correlación más generalizada de la geometría de la cavidad como función de las variables de proceso, se realizó un análisis basado en los principales grupos adimensionales que gobiernan la formación de la cavidad, esto es, los números de Froude $(F r)$, Weber $(W e)$, y Reynolds $(R e)$, que fueron considerados, después de un análisis dimensional de la fluidinámica del sistema chorro-baño líquido.

El análisis produjo las siguientes correlaciones para el ancho adimensional de la cavidad $(a / z)$ y la profundidad, también adimensional $(\mathrm{Hj} / \mathrm{z})$, como función, únicamente, de los números de Weber y Froude, ecuaciones $(6)$ y $(7)$ :

$$
\begin{gathered}
\frac{a}{z}=0,3^{*} W e^{1 / 4}\left(r^{2}=0,975\right) \\
\frac{H j}{z}=0,082^{*} \frac{W e^{1 / 2}}{F r^{5 / 29}}\left(r^{2}=0,995\right)
\end{gathered}
$$

Es interesante notar que el ancho de la cavidad es solo función del número de Weber; por tanto, este ancho depende solo de las interacciones entre las fuerzas inerciales del chorro gaseoso y la tensión superficial de la interfase. Por otro lado, la profundidad de la cavidad es función de los números de Froude y Weber, indicando que esta dimensión es controlada por el balance entre las fuerzas inerciales del chorro, las fuerzas boyantes del líquido y la fuerza de tensión superficial. Estas relaciones, basadas en balances de fuerzas para describir la deformación de la superficie libre, han sido discutidas por varios autores ${ }^{[5,8,14}$ y 15$]$. En nuestro sistema, es aparente que el número de Weber juega un papel más importante que el número de Froude, indicando que las fuerzas inerciales del chorro deben vencer, en primer término, las fuerzas de tensión superficial y, en segundo término, las fuerzas boyantes, para poder formar la profundidad de la cavidad. Los coeficientes de correlación para las ecuaciones (6) y (7) son 0,975 y 0,995, respectivamente.

La figura 7 muestra las mediciones experimentales del ancho (Fig. 7 a)) y la profundidad (Fig. 7b)) contra las predicciones de las ecuaciones (6) y (7) (línea sólida). El comportamiento predicho por las ecuaciones (6) y (7) es concordante con las mediciones experimentales, dada la pequeña dispersión de los datos experimentales.

Adicionalmente, los resultados experimentales obtenidos en este trabajo se contrastaron contra la correlación empírica reportada por Wakelin y Bradshaw ${ }^{[1]}$, que describe la profundidad adimensional de la cavidad como función de la cantidad de 


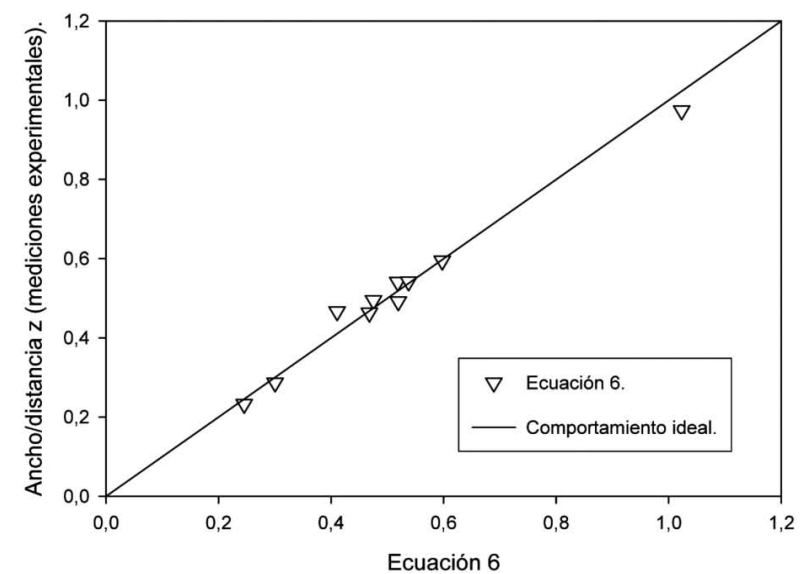

a)

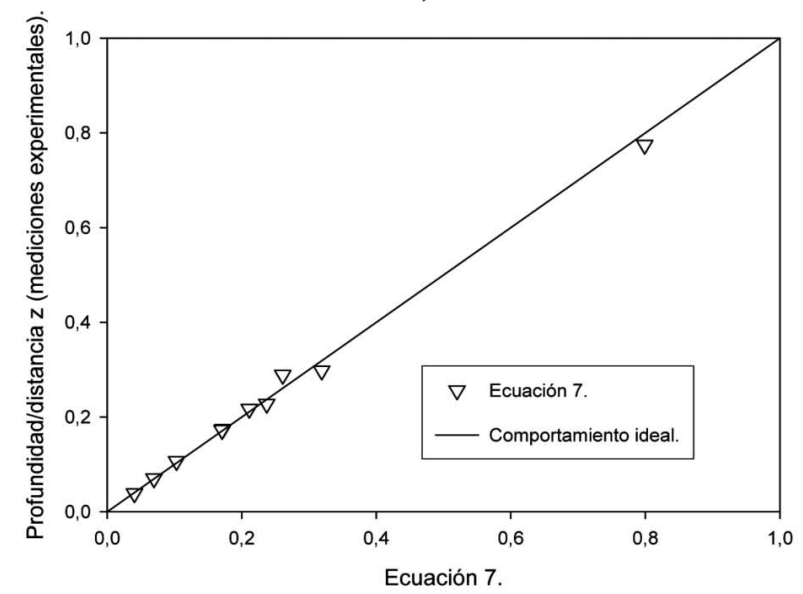

b)

Figura 7. Correlaciones empíricas obtenidas en este trabajo que describen: (a) el ancho adimensional de la cavidad como función del Número de Weber y (b) la profundidad adimensional como función de los números de Weber y Froude.

Figure 7. Empirical correlations obtained in this work to describe: (a) the dimensionless width of the cavity as a function of the Weber number and (b) the dimensionless cavity depth as a function of Weber and Froude numbers.

movimiento del chorro, la constante gravitacional, la densidad del líquido y la longitud, $z$, ecuación (8).

$$
\left(\frac{h}{z}\right)\left(\frac{h+z}{z}\right)^{2}=K\left(\frac{J z}{g^{*} \rho_{l}{ }^{*} z^{3}}\right)^{n}
$$

Donde $h$, es la profundidad de la cavidad, $z$ es la longitud $z, J z$ es la rapidez de transferencia de cantidad de movimiento, $g$ es la constante gravitacional, mientras que $K$ y $n$ son constantes a determinar. Las mediciones realizadas en este trabajo se representan gráficamente en la figura 8 junto con la ecuación (8)

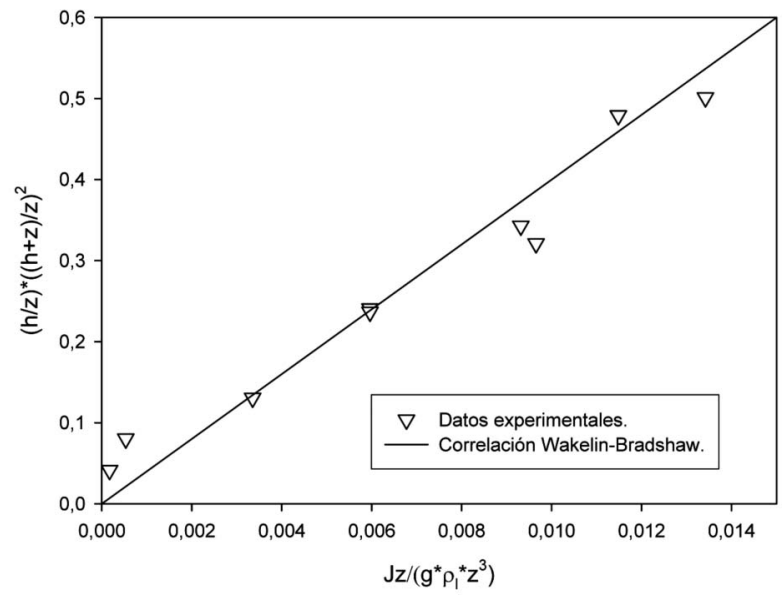

Figura 8. Resultados experimentales de la profundidad de la cavidad de acuerdo con la correlación empírica de Wakelin ${ }^{[1]}$.

Figure 8. Experimental results on cavity depth plotted according to the empirical correlation by Wakelin[1].

y se observa que la tendencia de los datos muestra una adecuada concordancia respecto a la correlación.

$\mathrm{Al}$ realizar el tratamiento estadístico de los datos obtenidos en este trabajo fue posible obtener una correlación similar a la de Wakelin, identificando el valor de $K$ de 3,63 y un exponente $n$, de 14/27, ecuación (9):

$$
\left(\frac{h}{z}\right)\left(\frac{h+z}{z}\right)^{2}=3.63\left(\frac{J z}{g^{*} \rho_{l}{ }^{*} z^{3}}\right)^{14 / 27}
$$

El coeficiente de correlación de esta expresión es 0,968 .

A manera de ilustración se presenta una extrapolación de las correlaciones obtenidas para la geometría de la cavidad con las condiciones experimentales en un convertidor BOF. Valores típicos del BOF incluyen: diámetro de boquilla de $0,057 \mathrm{~m}$, flujo volumínico de gas de $4,67 \mathrm{~m}^{3} / \mathrm{s}$ y altura de lanza de 1,75 $\mathrm{m}, \mathrm{a} 90^{\circ}$. Con éstos valores y aplicando la ecuación (7), se obtiene una profundidad de depresión estimada en el acero de 0,367 $\mathrm{m}$ y el valor hallado en la literatura a través de un nomograma ${ }^{[16]}$ es de 0,350 $\mathrm{m}$. Los números adimensionales, para este caso, son: $W e=803,14$ y $F r=975477,68$.

\subsection{Perfiles de velocidad}

Los perfiles promedio de velocidad obtenidos por PIV en la zona del baño afectada por la acción del chorro 
se presentan en las figuras $9,10,11$, y 12 bajo diferentes condiciones de proceso.

A partir de los resultados mostrados en estas figuras, se puede establecer que las velocidades se incrementan con el flujo de aire del chorro y decrecen con la distancia de la tobera al baño.

La figura 9 muestra el mapa vectorial de velocidades promedio en la región donde el chorro de aire incide en la superficie. La secuencia de imágenes (Figs. 9 b-e)) muestra la influencia del incremento en el flujo de gas. Las velocidades se incrementan al incrementarse el flujo de aire y la dirección del movimiento es causada por la dirección del flujo de gas, determinada por la posición de la lanza y su ángulo (todos estos casos se realizaron a una altura de lanza de $0,05 \mathrm{~m}$, ángulo de lanza de $60^{\circ}$ y $1,58 \times 10^{-3} \mathrm{~m}$ de diámetro de tobera). En esta figura se puede apreciar, claramente, que tanto el ancho como la profundidad de la cavidad crecen, al igual que la cantidad de fluido afectado por la transferencia de cantidad de movimiento del chorro (penetración del chorro) también aumenta con el incremento de flujo del chorro. Las velocidades máximas de 0,039 y $0,044 \mathrm{~m} / \mathrm{s} \mathrm{se} \mathrm{mi-}$ dieron bajo condiciones de flujo de aire de $1,6 \times 10^{-3}$ y $2 \times 10^{-3} \mathrm{~m}^{3} / \mathrm{s}$, respectivamente.

La figura 10 muestra el campo promedio de velocidades obtenido, variando el ángulo de la lanza y manteniendo constantes el resto de las condiciones (altura de lanza de 0,05 m, flujo de gas de $1,3 \times 10^{-3}$ $\mathrm{m}^{3} / \mathrm{s}$ y diámetro de tobera de $1,58 \times 10^{-3} \mathrm{~m}$ ). Conforme el ángulo de la lanza se desvía de la vertical, los vectores de velocidad del líquido muestran una mayor componente horizontal debido a que el esfuerzo cortante se incrementa conforme la lanza se acerca a una posición horizontal; la profundidad de la cavidad disminuye porque la componente normal de la cantidad de movimiento o la presión de incidencia del chorro disminuye conforme la lanza se orienta a una posición más cercana a la horizontal. Una lanza vertical $\left(90^{\circ}\right)$, bajo las mismas condiciones de

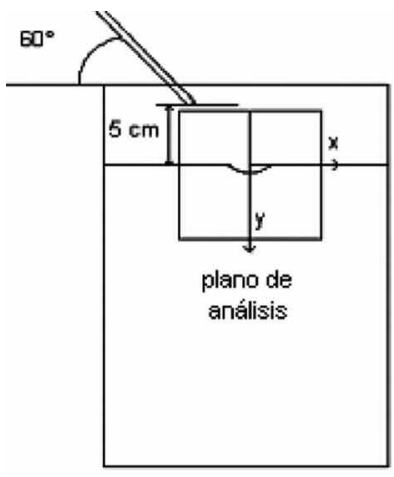

a)

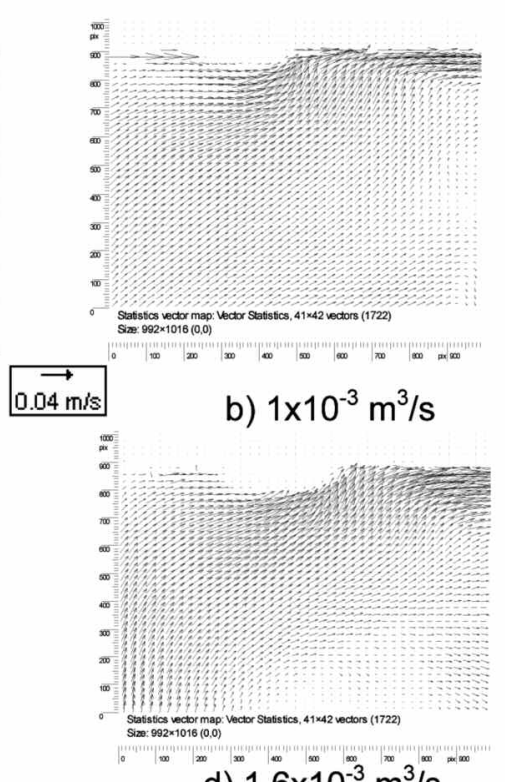

d) $1.6 \times 10^{-3} \mathrm{~m}^{3} / \mathrm{s}$

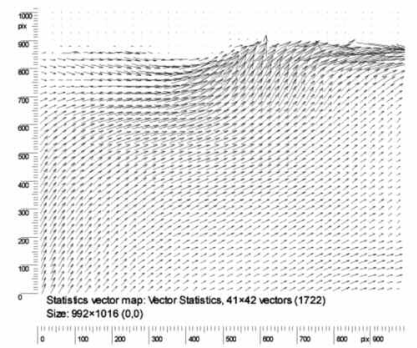

c) $1.3 \times 10^{-3} \mathrm{~m}^{3} / \mathrm{s}$

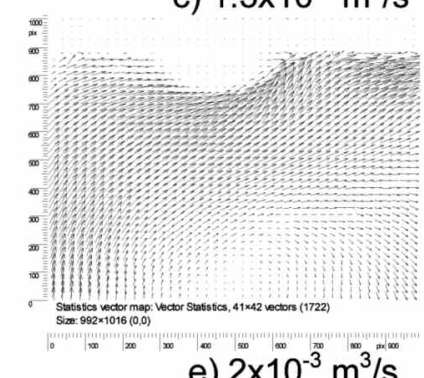

e) $2 \times 10^{-3} \mathrm{~m}^{3 / \mathrm{s}}$

Figura 9. Perfiles de velocidad promedio obtenidos por del PIV a diferentes flujos de gas: b) $1 \times 10^{-3} \mathrm{~m}^{3} / \mathrm{s}$; c) $1,3 \times 10^{-3} \mathrm{~m}^{3} / \mathrm{s}$; d) $1,6 \times 10^{-3} \mathrm{~m}^{3} / \mathrm{s}$ y e) $2 \times 10^{-3} \mathrm{~m}^{3} / \mathrm{s}$. El resto de las variables son: altura de lanza de $50 \mathrm{~mm}$, ángulo de lanza de $60^{\circ}$ y $1,58 \times 10^{-3}$ $\mathrm{m}$ de diámetro de tobera. El esquema de la izquierda, a), muestra la zona de analizada con el PIV, marcando el plano de análisis.

Figure 9. Time averaged liquid velocity vector profiles obtained by PIV with different gas flow rates: b) $1 \times 10^{-3} \mathrm{~m}^{3} / \mathrm{s}$; c) $1.3 \times 10^{-3} \mathrm{~m}^{3} / \mathrm{s}$; d) $1.6 \times 10^{-3} \mathrm{~m}^{3} / \mathrm{s}$ and e) $2 \times 10^{-3} \mathrm{~m}^{3} / \mathrm{s}$. The rest of variables are: lance height $0.05 \mathrm{~m}$, lance angle $60^{\circ}$ and $1.58 \times 10^{-3} \mathrm{~m}$ nozzle diameter. The left scheme, a), indicates the zone of PIV analysis showing a plane for flow measurements. 


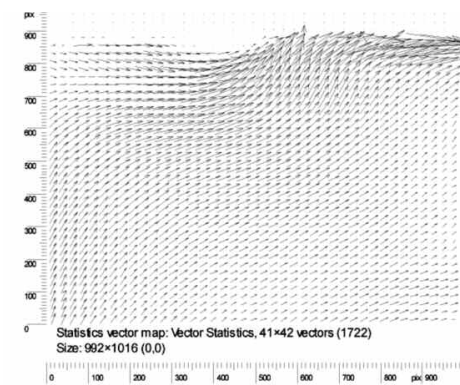

a) $60^{\circ}$

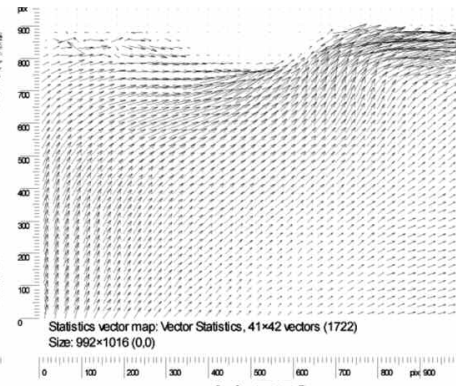

b) $75^{\circ}$

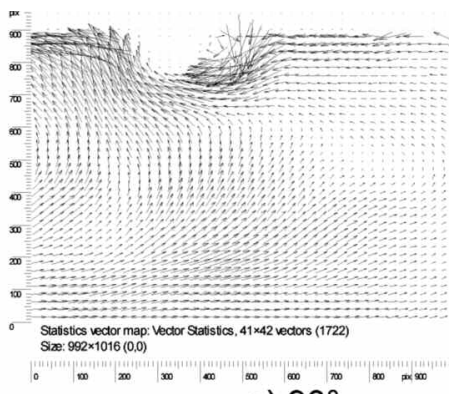

c) $90^{\circ}$

Figura 10. Perfiles de velocidad promedio obtenidos por PIV a diferentes ángulos de lanza: a) $60^{\circ}$; b) $75^{\circ}$ y c) $90^{\circ}$. El resto de las variables son: altura de lanza de $0,05 \mathrm{~m}$, flujo de gas de $1,3 \times 10^{-3} \mathrm{~m}^{3} / \mathrm{s}$ y $1,58 \times 10^{-3} \mathrm{~m}$ de diámetro de tobera.

Figure 10. Time averaged liquid velocity vector profiles obtained by PIV with different lance angles: a) $60^{\circ}$;b) $75^{\circ}$ and c) $90^{\circ}$. The rest of variables are: lance height $0.05 \mathrm{~m}$, gas flow rate $1.3 \times 10^{-3} \mathrm{~m}^{3} / \mathrm{s}$ and $1.58 \times 10^{-3} \mathrm{~m}$ nozzle diameter.

proceso, promueve una depresión de profundidad máxima. Una combinación de una pequeña distancia y un flujo de gas elevado dan como resultado una cavidad de 0,0178 $\mathrm{m}$, la profundidad máxima obtenida.

La figura 11 muestra los vectores de velocidad a diferentes diámetros de tobera y manteniendo constantes el resto de las variables (altura de lanza de $0,05 \mathrm{~m}$, flujo de gas de $1,3 \times 10^{-3} \mathrm{~m}^{3} / \mathrm{s}$ y ángulo de lanza de $60^{\circ}$ ). El tamaño de la tobera no afecta, de manera significativa, el intercambio de la cantidad de movimiento entre el chorro y la superficie del baño ya que las velocidades del chorro son, aproximadamente, las mismas. El cambio, el diámetro de tobera no influencia, significativamente, la cantidad de movimiento del chorro, bajo las condiciones del presente estudio.

La figura 12 muestra las velocidades del líquido obtenidas bajo diferentes alturas de lanza y manteniendo constantes el resto de las condiciones (ángulo de lanza de $60^{\circ}$, flujo de gas de $1,3 \times 10^{-3} \mathrm{~m}^{3} / \mathrm{s}, \mathrm{y}$ diámetro de tobera de $1,58 \times 10^{-3} \mathrm{~m}$ ). Las velocidades

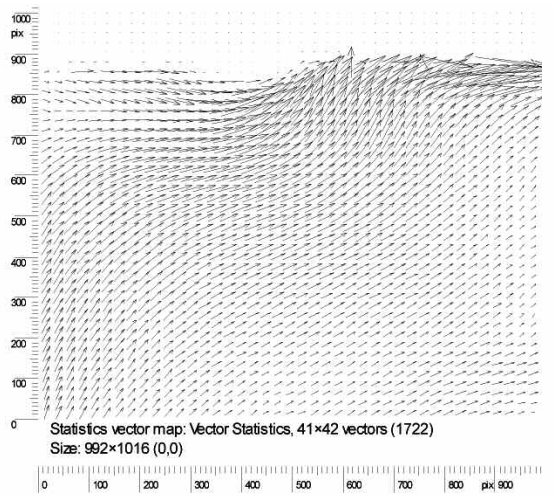

a) Diámetro de $1.58 \times 10^{-3} \mathrm{~m}$

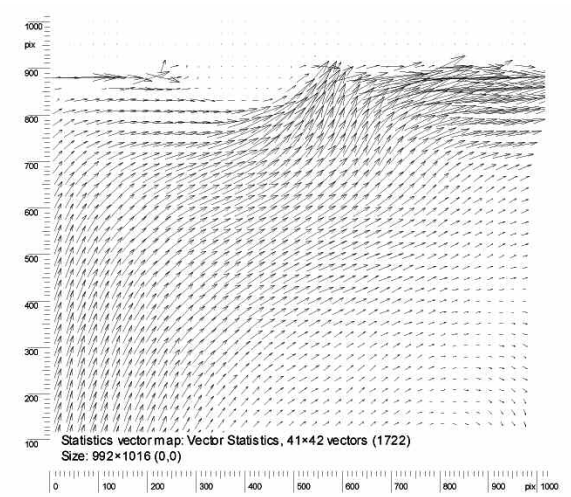

b) Diámetro de $3.17 \times 10^{-3} \mathrm{~m}$

Figura 11. Perfiles de velocidad promedio obtenidos por PIV a diferentes diámetros de tobera: a) $1,58 \times 10^{-3} \mathrm{~m}$ y b) $3,17 \times 10^{-3} \mathrm{~m}$. El resto de las variables son: altura de lanza de $0,05 \mathrm{~m}$, ángulo de lanza de $60^{\circ}$ y flujo de gas de $1,3 \times 10^{-3} \mathrm{~m}^{3} / \mathrm{s}$.

Figure 11. Time averaged liquid velocity vector profiles obtained by PIV with different nozzle diameters: a) $1.58 \times 10^{-3} \mathrm{~m}$ and b) $3.17 \times 10^{-3} \mathrm{~m}$. The rest of variables son lance height $0.05 \mathrm{~m}$, lance angle $60^{\circ}$ and gas flow rate of $1.3 \times 10^{-3} \mathrm{~m}^{3} / \mathrm{s}$. 


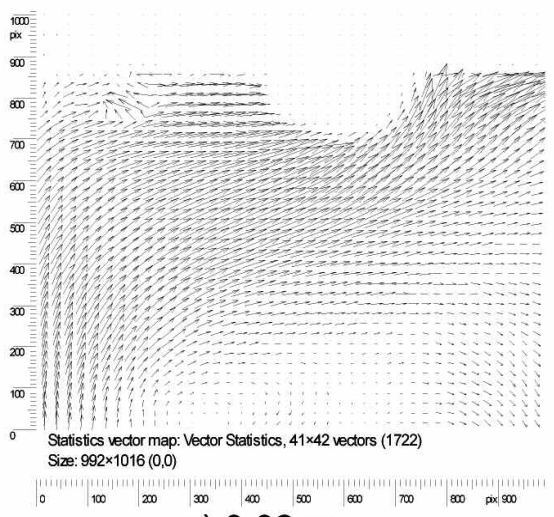

a) $0.02 \mathrm{~m}$

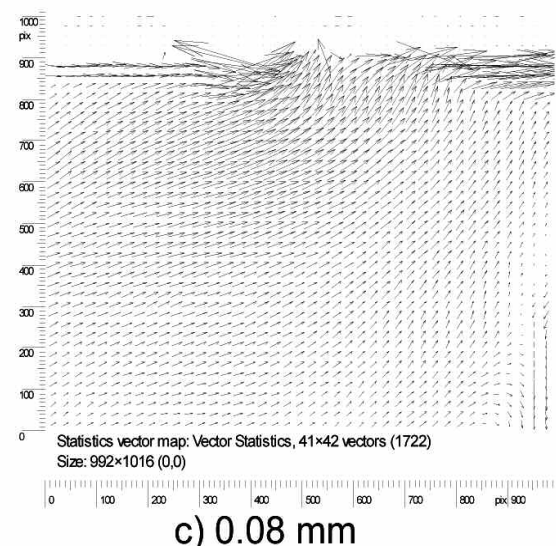

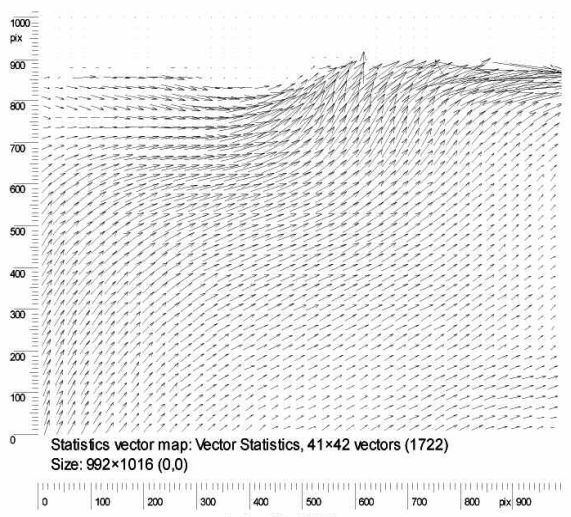

b) $0.05 \mathrm{~m}$

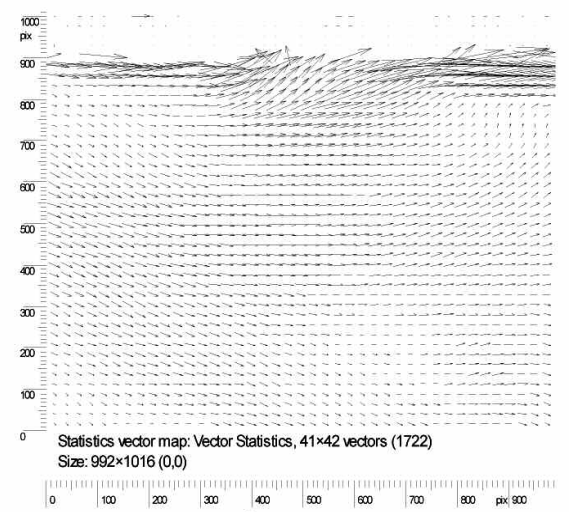

d) $0.1 \mathrm{~mm}$

Figura 12. Perfiles de velocidad promedio obtenidos por PIV a diferentes alturas de lanza: a) 0,02 m; b) 0,05 m; c) 0,08 m y d) 0,1 m. El resto de las variables son: ángulo de lanza de $60^{\circ}$, flujo de gas de $1,3 \times 10^{-3}$ $\mathrm{m}^{3} / \mathrm{s}$ y diámetro de lanza de $1,58 \times 10^{-3} \mathrm{~m}$.

Figure 12. Time averaged liquid velocity vector profiles obtained by PIV with different lance heights : a) $0.02 \mathrm{~m} \mathrm{b)} 0.05 \mathrm{~m}$; c) $0.08 \mathrm{~m}$ and d) 0.1 $\mathrm{m}$. The rest of variables are: lance angle $60^{\circ}$, gas flow rate of $1.3 \times 10^{-3}$ $\mathrm{m}^{3} / \mathrm{s}$ and $1.58 \times 10^{-3} \mathrm{~m}$ nozzle diameter.

medidas en el baño liquido y el tamaño de la cavidad se incrementan conforme las distancias entre la tobera y la superficie líquida disminuyen, debido a que la cantidad de movimiento del chorro se incrementa y tanto el esfuerzo cortante transmitido desde el chorro a la superficie del baño como la presión de incidencia se incrementan.

Para obtener información cuantitativa de la cantidad de movimiento transportado desde el chorro hacia el baño, las componentes en "X" de las velocidades obtenidas fueron representadas como función de la profundidad del baño, considerando, únicamente, una posición horizontal "X". La posición se muestra en la figura 9 a), que corresponde al punto en el cual el chorro incide en la superficie. Los perfiles de velocidad obtenidos a diferentes flujos de aire se presentan en la figura 13 a). Las velocidades en el baño aumentan, por debajo de la superficie libre, a causa de la cantidad de movimiento transferido por el jet, alcanzan un máximo, que es más profundo según incrementa el flujo $y$, entonces, las velocidades decrecen, poco a poco, conforme incrementa la profundidad. A $2 \times 10^{-3} \mathrm{~m}^{3} / \mathrm{s}$, se midió una velocidad máxima de $0,0338 \mathrm{~m} / \mathrm{s}$, a 0,0099 m por debajo de la cavidad. La figura $13 \mathrm{~b}$ ) muestra la componente en "X" de los perfiles de velocidad representada, con respecto a la profundidad para diferentes distancias verticales entre la tobera y la superficie libre. Conforme la tobera se aleja de la superficie libre, las velocidades disminuyen, pero el cambio en la posición de la tobera de 0,02 a 0,05 m parece mejorar la transferencia de la cantidad de movimiento en el baño, probablemente, porque la posición más cercana promueve pérdidas de cantidad de movimiento debido a las salpicaduras. Se alcanzó una 


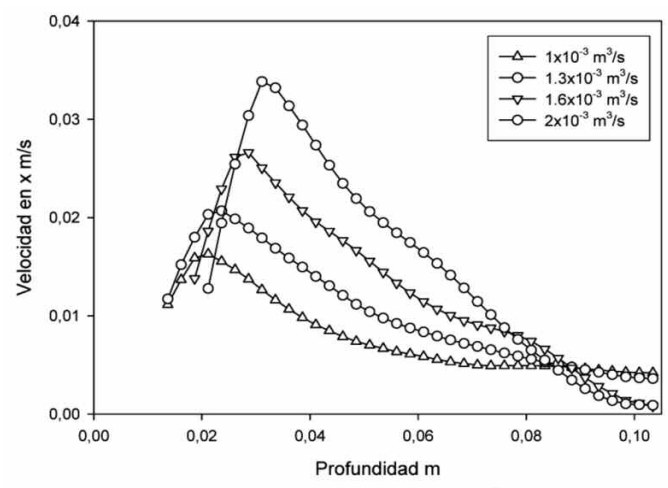

a)

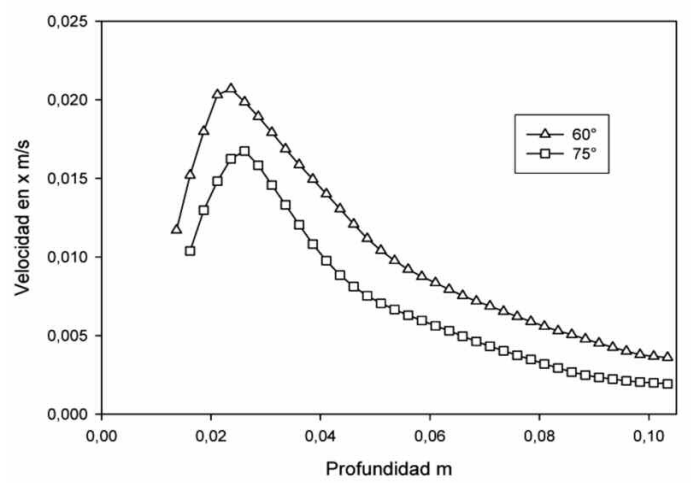

c)

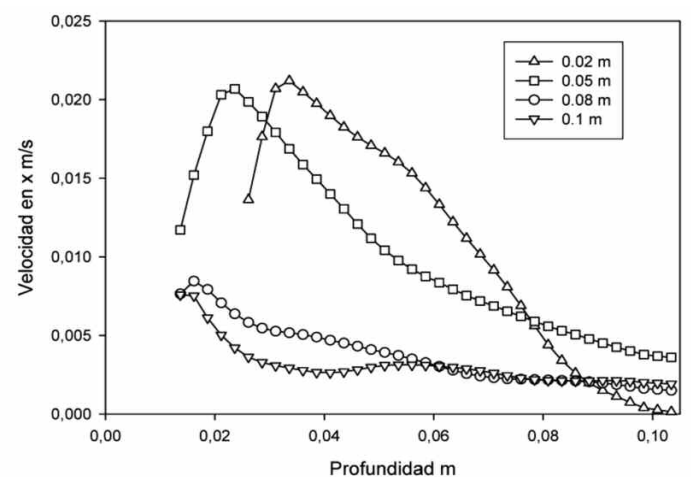

b)

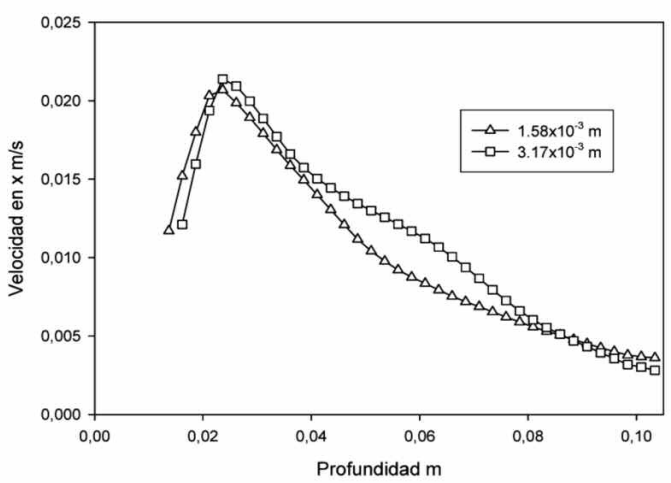

d)

Figura 13. Componente horizontal de las velocidades representado en función de la profundidad del baño: a) variando el flujo de gas y manteniendo el diámetro de tobera de $1,58 \times 10^{-3} \mathrm{~m}$, ángulo de lanza de $60^{\circ}$ y altura de lanza $(h)$ de $0,05 \mathrm{~m}$; b) variando altura de lanza, y con diámetro de tobera de $1,58 \times 10^{-3} \mathrm{~m}$, flujo de gas de $1,3 \times 10^{-3} \mathrm{~m}^{3} / \mathrm{s}$, ángulo de lanza de $60^{\circ}$; c) variando el ángulo de lanza y flujo de gas de $1,3 \times 10^{-3} \mathrm{~m}^{3} / \mathrm{s}$, diámetro de tobera de $1,58 \times 10^{-3} \mathrm{~m}$ y altura de lanza $(h)$ de 0,05 $\mathrm{m}$; d) variando diámetro de tobera, y flujo de gas de $1,3 \times 10^{-3} \mathrm{~m}^{3} / \mathrm{s}$, ángulo de lanza de $60^{\circ}$ y altura de lanza (h) de $0,05 \mathrm{~m}$.

Figure 13. Horizontal component of liquid velocity plotted as function of the depth of the bath: a) varying gas flow rate, other variables are nozzle diameter $1.58 \times 10^{-3} \mathrm{~m}$, lance angle of $60^{\circ}$ and lance height (h) $0.05 \mathrm{~m}$; b) varying lance height, other variables are nozzle diameter $1.58 \times 10^{-3} \mathrm{~m}$, gas flow rate $1.3 \times 10^{-3} \mathrm{~m}^{3} / \mathrm{s}$, and lance angle $60^{\circ}$; c) varying lance angle, other variables are gas flow rate $1.3 \times 10^{-3} \mathrm{~m}^{3} / \mathrm{s}$, nozzle diameter $1.58 \times 10^{-3} \mathrm{~m}$ and lance height (h) $0.05 \mathrm{~m}$; d) varying Nozzle diameter, other variables gas flow rate $1.3 \times 10^{-3} \mathrm{~m}^{3} / \mathrm{s}$, lance angle $60^{\circ}$ and lance height (h) $0.05 \mathrm{~m}$.

velocidad máxima de $0,0212 \mathrm{~m} / \mathrm{s}$, para una altura de lanza de $0,02 \mathrm{~m}$, esto es, $0,0074 \mathrm{~m}$ por debajo de la cavidad. El efecto del ángulo de lanza se muestra en la figura 13 c). Las velocidades son mayores para un ángulo de lanza de $60^{\circ}$ que para uno de $75^{\circ}$, debido a que el la componente en "X" de la cantidad de movimiento se transfiere mejor para un ángulo más cercano a la horizontal. Un valor máximo de $0,0207 \mathrm{~m} / \mathrm{s}$ se obtuvo, a 0,01 m de la cavidad. El diámetro de la tobera no tiene efecto en los perfiles de velocidad ya que las gráficas que se muestran en la figura $13 \mathrm{~d}$ ) pa- ra diámetros de tobera de $1,58 \times 10^{-3}$ y $3,17 \times 10^{-3} \mathrm{~m}$ son similares.

El flujo volumínico de líquido, causado por el esfuerzo cortante proveniente del chorro, es un parámetro importante que indica la capacidad del chorro para inducir el movimiento en la masa líquida del baño. El flujo del líquido del baño se calculó integrando numéricamente el perfil de velocidades que se presenta en la figura 13 con la profundidad del baño y usando el método del trapecio, ecuación (10). 


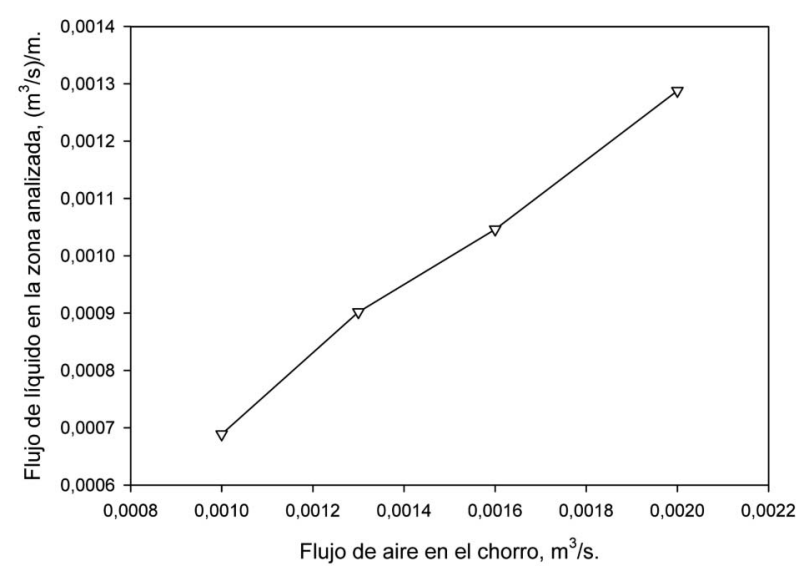

Figura 14. Flujo de agua como función del flujo de aire del chorro.

Figure 14. Water flow rate as a function of jet air flow rate.

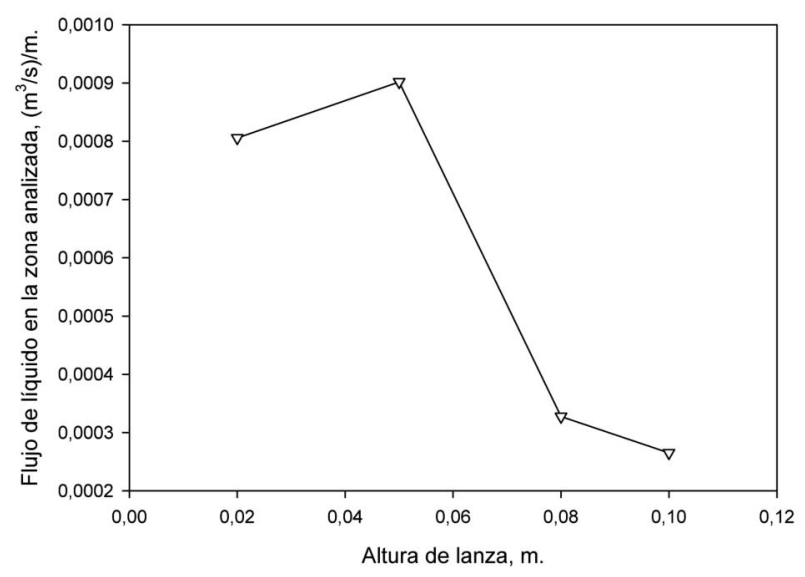

Figura 15. Flujo de agua como función de la altura vertical de lanza.

Figure 15. Water flow rate as a function of nozzle vertical height.

$$
Q_{1}=\int_{0}^{H} V_{x} d y
$$

Naturalmente, el valor de tiene unidades de flujo volumínico por unidad de longitud visual $\left(\mathrm{m}^{3} / \mathrm{s}\right) / \mathrm{m}$. La figura 14 indica que el flujo de líquido se incrementa linealmente con el incremento de flujo de aire, presentando un máximo de $1,3 \times 10^{-3} \mathrm{~m}^{2} / \mathrm{s}$ a $2 \times 10^{-3} \mathrm{~m}^{3} / \mathrm{s}$; mientras que la figura 15 muestra que un incremento en la distancia entre la tobera y la superficie libre es inversamente proporcional al flujo del baño causado por la incidencia del chorro. Un valor máximo del flujo de $9,02 \times 10^{-4} \mathrm{~m}^{2} / \mathrm{s}$ se obtuvo, a 0,05 $\mathrm{m}$ de altura de lanza y no en la altura mínima de $0,02 \mathrm{~m}$. Esto se puede explicar si se toma en consideración que parte de la cantidad de movimiento del chorro se pierde a causa del fenómeno de salpicado que ocurre en esta distancia mínima entre la tobera y la superficie libre $(0,02 \mathrm{~m})$.

\section{CONCLUSIONES}

- El flujo de líquido causado por el esfuerzo cortante del chorro gaseoso aumenta linealmente conforme disminuye el flujo de gas.

- El flujo de líquido, causado por el esfuerzo cortante del chorro gaseoso como función de la altura de lanza, presenta un máximo a cierta distancia y, después, decrece conforme se incrementa la altura de lanza, porque la cantidad de movimiento del chorro en la superficie libre disminuye conforme la lanza se aleja de la superficie libre. Sin embargo, a distancias cortas, el flujo es pequeño, presumiblemente, debido a las pérdidas derivadas del salpicado.

- El diámetro de tobera no tiene un efecto importante en el flujo causado por la incidencia del chorro, porque los diámetros de tobera usados en este trabajo no afectan los perfiles de velocidad del chorro y la cantidad de movimiento del chorro de manera significativa.

- El flujo de líquido causado por el esfuerzo cortante del chorro como función de los ángulos de lanza es alto cuando el ángulo de lanza es cercano a la horizontal, ya que el componente horizontal de la velocidad es mayor y, en consecuencia, el esfuerzo cortante transmitido del gas al líquido se incrementa.

- El ancho de la cavidad es función, únicamente, del número de Weber de acuerdo a la correlación obtenida en este trabajo. El valor del coeficiente en la correlación empírica indica que las fuerzas inerciales predominan sobre las fuerzas de tensión superficial, en cuanto al ancho de la cavidad.

- La correlación empírica para la profundidad de la cavidad muestra que las fuerzas inerciales afectan más el tamaño de la profundidad de la cavidad que la fuerza de tensión superficial y las fuerzas boyantes.

- Las mejores condiciones de operación (con los valores usados en este trabajo) en términos del flujo de líquido causado por la transferencia de cantidad del movimiento del chorro gaseoso al baño líquido son: el mayor flujo de aire $\left(2 \times 10^{-3} \mathrm{~m}^{3} / \mathrm{s}\right)$ y una altura de lanza de $0,05 \mathrm{~m}$ puesto que, con estas condiciones, la cantidad de movimiento se transfiere más eficientemente del 
chorro al baño. A una menor altura de lanza, el salpicado disminuye la transferencia de la cantidad de movimiento del chorro al baño.

\section{Agradecimientos}

Los autores desean expresar su agradecimiento al Consejo Nacional de Ciencia y Tecnología (CONACYT) de México por el soporte financiero brindado, por medio del proyecto 60033 y por la proyecto doctoral otorgada a J. S.-L. También se agradece el apoyo financiero brindado por parte de la DGAPA a través del proyecto PAPIIT IN109310. Adicionalmente, desean agradecer al Profesor Adrián M. Amaro V. por su inestimable ayuda en la parte experimental de este trabajo.

\section{Lista de símbolos}

a, ancho o diámetro de la cavidad.

$d_{c}, d_{e}, d_{t}^{\prime}, d_{t}$, diámetros de tobera.

$f$, flujo de aire en $\mathrm{m}^{3} / \mathrm{s}$.

Fr, número de Froude.

$\mathrm{Fr}_{m}^{\prime}$, número de Froude modificado.

$g$, constante gravitacional.

$h, h^{\prime}$, altura de lanza.

$\mathrm{Hj}, \mathrm{Hj}^{\prime}$, profundidad de la cavidad.

$J$, tasa de transferencia de la cantidad de movimiento del chorro.

$K$, constante adimensional.

$L$, longitud característica.

$P$, presión.

$p_{a m b}$, presión atmosférica.

$p_{o}$, presión del chorro.

$\mathrm{Q}_{l}$, flujo del líquido por unidad de profundidad.

$R_{0}$, diámetro de curvatura de la depresión.

$V_{\mathrm{g}}$, velocidad de gas.

$V_{g}^{\prime \prime}$, velocidad de gas desarrollada.

$V_{x}$, componente en $\mathrm{x}$ de la velocidad.

We, número de Weber.

$z$, distancia en $\mathrm{m}$.

$a$, ángulo de lanza. $\mu$, viscosidad.

$r_{l}$, densidad de líquido.

$r_{g}$, densidad de gas.

$\stackrel{g}{\sigma}$, tensión superficial.

\section{REFERENCIAS}

[1] D. H. Wakelin, Tesis doctoral, University of London, 1966.

[2] M. Lee, V. Whitney and N. Molloy: Scand. J. Metall. 30 (2001) 330-336.

[3] S. K. Sharma, J. W. Hlinka and D. W. Kern, Ironmak. Steelmak. 4 (1977) 7-18.

[4] A. Nordqist, N. Kumbhat, L. Jonsson and P. Jonssön, Steel Res. 77 (2006) 82-90.

[5] S. C. Koria and K. W. Lange, Steel Res. 589(1987) 421-426.

[6] F. Qian, R. Muthasaran and B. Farouk: Metall. Mat. Trans. B 27 (1996) 911-920.

[7] G. A. Subagyo, K. S. Brooks, Coley, G. A. Irons, ISIJ Int. 43 (2003) 983-989.

[8] F. Memoli, C. Mapelli, P. Ravanelli, M. Corbella, ISIJ Int. 44 (2004) 1.342-1.349.

[9] A. R. N. Meidani, M. Isac, A. Richardson, A. Cameron and R. I. L. Guthrie: ISIJ Int. 44 (2004) 1639-1645.

[10] Y. Tago and Y. Higuchi, ISIJ Int. 43 (2003) 209 215.

[11] L. Gu, G. Irons, Electric Furnace Conf. Proc. 1999, pp. 269-278.

[12] M. P. Schwarz, Fluid Flow Phenomena in Metals Processing, The Minerals, Metals \& Materials Society, 1999, pp. 171-178.

[13] K. D. Peaslee, D. G. C. Robertson, The Minerals, Metals \& Materials Society. EPD Congress, 1994.

[14] R. B. Banks, D. V. Chandrasekhara, J. Fluid Mech. 15 (1963) 13-34.

[15] P. Mc Gee, G. A.Irons, Electric Furnace Conf. Proc., 1999, pp. 439-446.

[16] J., Szekely, Fenómenos de flujo de fluídos en el procesamiento de metales., $1^{a}$. Edición, Editorial Limusa, México, 1988, pp. 423-424. 